\title{
Bone-Targeted Extracellular Vesicles from Mesenchymal Stem Cells for Osteoporosis Therapy
}

This article was published in the following Dove Press journal: International Journal of Nanomedicine

\author{
Yayu Wang ${ }^{1, *}$ \\ Jie $\mathrm{Yao}^{2-4, *}$ \\ Lizhao $\mathrm{Cai}^{2-4, *}$ \\ Tong Liu' \\ Xiaogang Wang ${ }^{2,4}$ \\ Ye Zhang ${ }^{2-4}$ \\ Zhiying Zhou ${ }^{2-4}$ \\ Tingwei $\mathrm{Li}^{2-4}$ \\ Minyi Liu ${ }^{2-4}$ \\ Renfa $\mathrm{Lai}^{2-4}$ \\ Xiangning $\mathrm{Liu}^{2-4}$ \\ 'Department of Cell Biology \& Institute \\ of Biomedicine, College of Life Science \\ and Technology, Jinan University, \\ Guangzhou 510632, People's Republic of \\ China; ${ }^{2}$ Department of Stomatology \\ Medical Center, The First Affiliated \\ Hospital of Jinan University, Guangzhou, \\ 5 I0630, People's Republic of China; \\ ${ }^{3}$ School of Stomatology, Jinan University, \\ Guangzhou 510632, People's Republic of \\ China; ${ }^{4}$ Clinical Research Platform for \\ Interdiscipline of Stomatology, Jinan \\ University, Guangzhou 510630, People's \\ Republic of China
}

*These authors contributed equally to this work

Correspondence: Xiangning Liu; Renfa Lai The First Affiliated Hospital of Jinan University, Guangzhou 510632, People's Republic of China

Tel +86203868 8109

Fax +862038688000

Email liuxiangning2003@।26.com;

prof.dr.lai@163.com
Background: Current drugs used for osteoporosis therapy show strong adverse effects. Stem cell-derived extracellular vesicles (EVs) provide another choice for osteoporosis therapy. Mouse mesenchymal stem cells (mMSCs)-derived EVs promote bone regeneration; however, their clinical application is limited due to non-specific tissue targeting. Alendronate specifically targets bone tissue via hydroxyapatite. Therefore, EVs were combined with alendronate to generate Ale-EVs by "click chemistry" to facilitate EVs targeting bone via alendronate/hydroxyapatite binding.

Methods: Ale-EVs were characterized based on size using dynamic light scattering analysis and morphology was visualized by transmission electron microscopy. Hydroxyapatite affinity of Ale-EVs was detected by flow cytometry. Bone targeting of Ale-EVs was tested by ex vivo fluorescent imaging. Cell viability was assessed by using WST-8 reduction assay kit for testing the ability of Ale-EVs to promote mMSCs proliferation. Alkaline phosphatase experiment was used to detect ability of Ale-EVs to promote differentiation of mouse mesenchymal stem cells in vitro. Western blotting and Q-PCR assay were used to detect the early marker of osteogenic differentiation. Antiosteoporotic effects of Ale-EVs were detected in ovariectomy (OVX)-induced osteoporosis rat model. The safety of the Ale-EVs in vivo was measured by H\&E staining and serum markers assay.

Results: In vitro, Ale-EVs had high affinity with hydroxyapatite. Also, ex vivo data indicated that Ale-EVs-DiD treatment of mice induced strong fluorescece in bone tissues compared with EVs-DiD group. Furthermore, results suggested that Ale-EVs promoted the growth and differentiation of mouse MSCs. They also protected against osteoporosis in ovariectomy (OVX)-induced osteoporotic rats. Ale-EVs were well tolerated and no side effects were found, indicating that Ale-EVs specifically target bone and can be used as a new therapeutic in osteoporosis treatment.

Conclusion: We used the Ale-N3 to modify mouse mesenchymal stem cells-derived extracellular vesicles by copper-free "click chemistry" to generate a Ale-EVs system. The AleEVs had a high affinity for bone and have great potential for clinical applications in osteoporosis therapy with low systemic toxicity.

Keywords: extracellular vesicles, EVs, mesenchymal stem cells, MSCs, bone-targeting, osteoporosis, click chemistry

\section{Background}

With the development of society and the increase of average life expectancy, osteoporosis (OP) has become one of the most common chronic diseases in today's society. ${ }^{1}$ Many studies have shown that OP can adversely affect the healing of 
mandible fractures and defects, and delay bone healing. ${ }^{2}$ Besides, OP is closely related to the effect of dental implant restoration. It has been reported that the bone resorption around implants in OP patients is significantly higher than those with normal bone density within five years after implantation and repair. Temmerman confirmed that the five-year survival rate of implants in OP patients is lower than that in the normal group. ${ }^{3-5}$ Therefore, effective treatment of OP can not only improve the quality and efficiency of bone healing in OP patients, but also shorten the time of osseointegration.

At present, the drugs of OP mainly include bone resorption inhibitors (such as bisphosphonates, estrogen, selective estrogen receptor modulators and calcitonin); bone formation promoter (such as parathyroid hormone analogs) and others (such as vitamin $\mathrm{K}$, active vitamin $\mathrm{D}$ and its analogs). ${ }^{6-10}$ Alendronate (Ale) is the most widely used first-line anti-OP drugs, it contains a P-C-P group of the pyrophosphate analog, which specifically binds to bone surface through a high affinity with hydroxyapatite to inhibit bone resorption by suppressing osteoclast function. ${ }^{11}$ However, the drug has side effects and may cause oesophagitis and gastrointestinal discomfort. $^{12}$ Therefore, it is very important to find more safe and effective drugs for OP. With the development of regenerative medicine, exosomes (extracellular vesicles, EVs) provide a new choice for OP therapy.

EVs can transport proteins and nucleic acids. They can be separated to apoptotic bodies, intermediate-sized microvesicles, and exosomes according to sizes. Exosomes are the most studied EVs that can be secreted by many types of cells. ${ }^{13-15}$ Exosomes participate in a variety of physiological and pathological processes, such as angiogenesis, tissue regeneration, and regulating wound healing by carrying biologically active molecules such as proteins and lipids, as well as mRNA and microRNA. ${ }^{16} \mathrm{Hu}$ 's team studied the effects of EVs in umbilical cord blood (UCB) for elderly OP, the results showed that intravenous injection of EVs from UCB into aged male $\mathrm{C} 57 \mathrm{BL} / 6$ mice for 2 months could reduce bone loss. Computed tomography (CT) results showed an increment of trabecular and cortex bone mass. Study showed that miR-3960, highly enriched in EVs of UCB, affects osteoblastic differentiation of bone marrow stem cells (BMSCs). ${ }^{17}$ There are also reports showed that mesenchymal stem cells (MSCs)-derived EVs had good potential for the treatment of OP. ${ }^{18}$ However, natural EVs do not specifically target bones, which greatly limits their clinical application. So, how to effectively use EVs to promote osteogenesis and improve their bioavailability is an important research direction.

Bone-targeting drug molecules target bone. However, it is difficult to couple these bone-targeted small molecules to the surface of drug carriers under normal conditions. As an efficient and convenient chemoselective conjugation method under gentle conditions, "click chemistry" can solve this problem. ${ }^{19}$ The "click chemical" reaction is mild, easy to operate and easy to purify products with no harmful by-products. It's commonly used in synthesis of functional polymers, cell markers, and biomedical synthesis. $^{20,21}$ Stefanucci Azzurra's team reported a new enkephalin analogue with great analgesic, which was constructed via "click chemistry", 22 Lee's team used copperfree "click chemistry" to combine growth factors with collagen. 23 "Click chemistry" is a powerful synthetic tool in the discovery of new drugs. ${ }^{24}$

In this study, we modified the Ale molecule with an azide group (N3), and modified the membrane surface of EVs with an alkynyl (DBCO) group using a commercial linker. Then, Ale-N3 and EVs-DBCO were coupled by "click chemistry". Finally, the Ale-EVs complex was constructed (Figure 1A). We then assessed the bone targeting ability of Ale-EVs. Results showed that Ale-EVs promoted growth and differentiation of mouse MSCs in vitro and protected against osteoporosis in vivo, suggesting that Ale-EVs could be a new therapeutic for osteoporosis.

\section{Materials and Methods Materials}

Dynabeads $^{\circledR}$ was obtained from Life Technologies; 2-(2-methoxy-4-nitrophenyl)-3-(4-nitrophenyl)-5-(2,4-disulfophenyl)-2H-tetrazolium, monosodium salt (WST-8) reduction assay kit was purchased from Dojindo Molecular Technologies; Anti-Col1 A1 (sc-293182), AntiRUNX-2 (sc-10758) were from Santa-Cruz; Alendronic acid (CAS: 66376-36-1) was purchased from Aladdin (China); $\mathrm{NaOH}$ was from Sigma Aldrich (USA); Acetonitrile (ACN, HPLC grade) was purchased from Tianjin Concord Technology Co., Ltd; azidobutyric acid NHS ester was purchased from Lumiprobe Corporation; DBCO-PEG4-NHS ester was from Click Chemistry Tools (USA); 
A
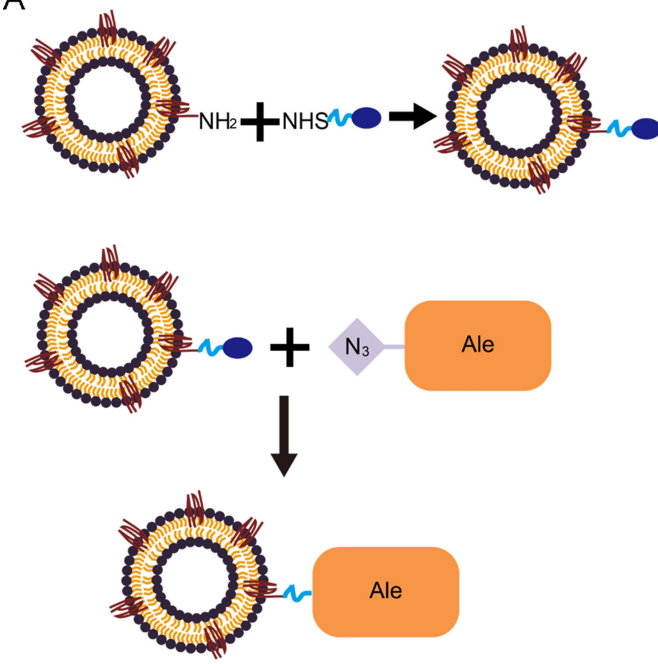

EVs $\equiv \underset{\text { protein }}{\operatorname{membrane}} \underset{\text { group }}{\mathrm{DBCO}} \sim$ linker

C

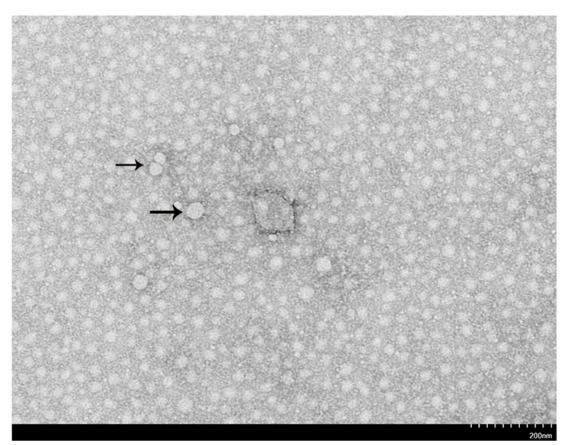

B

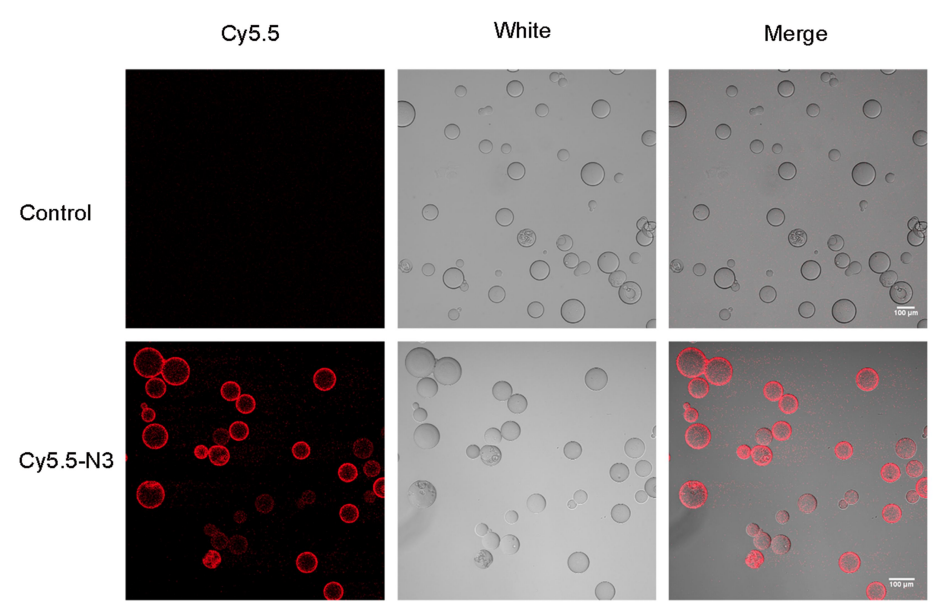

D

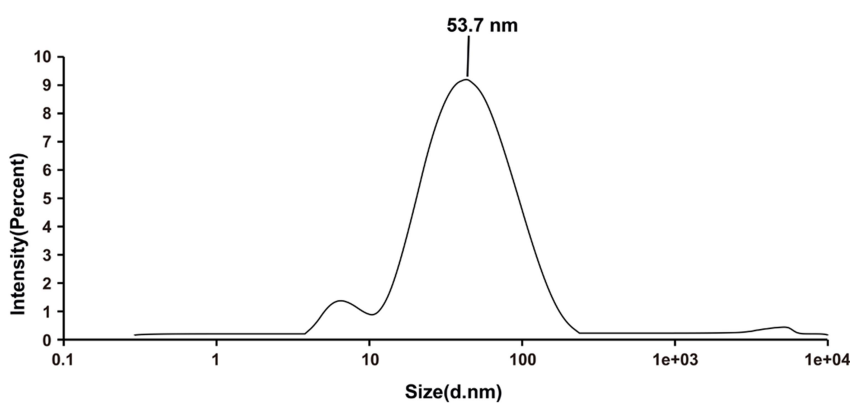

Figure I Ale-EVs generation and characterization. (A) Synthesis of Ale-EVs. (B) Analysis of the N3-Cy5.5 conjugation with EVs. EVs were conjugation with N3-Cy5.5 via "Click Chemistry". Cy5.5-EVs were captured by Dynabeads and fluorescence was analyzed. (C) TEM observation of Ale-EVs showed intact (30-200 nm) (Arrows: Ale-EVS). (D) Size distribution of Ale-EVs analyzed by DLS. The peak diameter was at $53.7 \mathrm{~nm}$.

Abbreviations: Ale, alendronate; EVs, extracellular vesicles; DLS, dynamic light scattering.

\section{Cells}

Mouse MCSs (mMSCs) were obtained from ATCC, and cultured in RPMI 1640 (Invitrogen, CA) with 10\% fetal bovine serum (FBS) (Invitrogen, CA) and Pen $/$ strep at $37^{\circ} \mathrm{C}$.

\section{Synthesis of Azido-Ale}

Alendronate disodium salt $(293 \mathrm{mg})$ was dissolved with PBS $(15 \mathrm{~mL})$. The aqueous solution was then cooled in an ice water bath, and mixed with a solution of azidobutyric acid NHS ester (226.2 MW) in acetonitrile (ACN) $(14 \mathrm{~mL}, 0.267 \mathrm{~mol})$. The mixture was kept at $25^{\circ} \mathrm{C}$ with stirring for 2 hours and directly purified with reversedphase high performance liquid chromatography (RPHPLC) (Waters XTERRA Prep MS C8 OBD column, 5 $\mu \mathrm{m}, 19 \times 150 \mathrm{~mm}$ ), flow rate $10 \mathrm{~mL} / \mathrm{min}, 214 \mathrm{~nm} \mathrm{UV}$.
Buffer A: $0.1 \%$ trifluoroacetic acid (TFA)/H2O. Buffer B: $0.1 \%$ TFA/ACN. Gradient: $100 \%$ buffer A, run $6 \mathrm{~min}$; $100 \%$ buffer B, $1 \mathrm{~min} ; 100 \%$ buffer B, 5 min. $100 \%$ buffer A, 1 min. 100\% buffer A, 5 min. Then the collected eluent was lyophilized to obtain a colorless (or off-white) solid product. The identity of the product was verified by Orbitrap ESI MS (negative mode): Found 359.05 (M-H)-. Calculated: $359.05(\mathrm{M}-\mathrm{H})$-.

\section{Isolation and Purification of EVs}

The mMSCs were cultured in RPMI1640 with 10\% FBS. FBS was centrifuged at $120,000 \mathrm{~g}$ for 2 hours to remove the EVs. Cell culture medium was collected and spun at $300 \mathrm{~g}$ for $15 \mathrm{~min}$ to discard the dead cells, impurities and other pellet. The mMSCs supernatant was centrifuged at 
$2000 \mathrm{~g}$ for $15 \mathrm{~min}$. The supernatant was collected and centrifuged at 10,000g for 2 hours. The supernatant was centrifuged at $100000 \mathrm{~g}$ for $70 \mathrm{~min}$ again. The supernatants were removed and EVs pellets were re-suspended in PBS.

\section{Synthesis and Characterization of Ale-EVs}

Ale-EVs were synthesized through conjugation of Ale-N3 and EVs-DBCO via copper catalyzed acetylene-azide cycloaddition reaction. First, DBCO-PEG4-NHS ester was used to add DBCO functional group to EVs' surface through a reaction of NHS ester with amine group (NH2) of EVs membrane protein. Freshly prepared alkaline PBS ( $\mathrm{pH}$ 8.8), and appropriate amount of DBCO-PEG4-NHS ester solution and EVs solution (molar ratio $=10: 1$ ) were incubated overnight at room temperature (RT) in the dark with rotation to form an EVs-DBCO compound. The EVs$\mathrm{DBCO}$ was placed in a $50 \mathrm{KD}$ ultrafiltration tube and centrifuged at $10000 \mathrm{~g}$ for 30 minutes. After centrifugation, -DBCO compounds were collected and diluted with PBS. Ale-N3 solution was added at a molar ratio of 10:1 to EVs, incubated overnight at RT in the dark with rotation. The solution was removed into the $50 \mathrm{KD}$ ultrafiltration tube and centrifuged at $10000 \mathrm{~g}$ for 30 minutes to remove the uncoupled small molecules and to obtain the Ale-EVs compounds.

\section{Transmission Electron Microscopy}

Ale-EVs $(15 \mu \mathrm{g})$ were fixed with $4 \%$ PFA for $30 \mathrm{~min}$ at RT, dispersed using ultrasonic bath for $2 \mathrm{~min}$, vortexed, dried on UV-treated grids, stained with uranyl acetate $(2 \%)$ for 15min, and observed by TEM (Philips Tecani 10, Netherlands).

\section{In vitro Bone Targeting of Ale-EVs}

Fluorescence staining was performed in Ale-EVs and EVs at RT in the dark. The two kinds of particles were mixed with DiD dye for 20 minutes. After incubation, the supernatant was placed in a $50 \mathrm{KD}$ ultrafiltration tube and centrifuged at $10000 \mathrm{~g}$ for $30 \mathrm{~min}$ to get Ale-EVs-DiD and EVs-DiD. Equal amount of Ale-EVs or EVs was combined with appropriate amount of hydroxyapatite beads for 2 hours. Then, these mixtures were centrifuged at 1500 $\mathrm{g}$ for $5 \mathrm{~min}$. Particles were re-suspended in PBS, centrifuged again at $1500 \mathrm{~g}$ for $5 \mathrm{~min}$, and re-suspended in 300 $\mu \mathrm{L}$ of PBS flow cytometry analysis.

\section{In vivo Bone Targeting of Ale-EVs}

Ale-EVs-DiD (150 $\mu$ g of EVs) was given to nude mice by tail vein injection. After 6 hours, DiD fluorescence was analyzed using In-Vivo Xtreme (Bruker, Germany) six hours later. DiD fluorescence in heart, liver, lung, spleen, kidney, intestine and bone tissues were also measured and analyzed using MI SE software (Bruker).

\section{ALP Staining Experiment}

Alkaline phosphatase (ALP) is an important indicator for detecting osteogenic differentiation of mMSCs. In this study, different types of mixtures were used to treat mMSCs plated on cell culture plates. The ALP staining was used to test whether different groups of Ale-EVs had the ability to induce osteogenic differentiation. mMSCs were lysed and centrifuged at 12,000 g for $10 \mathrm{~min}$, followed by ALP activity assay (Beyotime, shanghai, China). After incubation at $37^{\circ} \mathrm{C}$ for 30 minutes, termination solution was added and the activity of ALP was measured at $405 \mathrm{~nm}$.

\section{Q-PCR Assay}

RNAs were extracted using Trizol (Invitrogen) and quantified using NanoDrop-2000 (Thermo, USA). cDNA was synthesized by SuperScript II kit (Invitrogen). SYBR Green Mastermix (ABI, USA) was used for Q-PCR. Primers were: 18s rRNA-Forward: GTAACCCGTTGAACCCCATT, and 18s-Reverse: CCATCCAATCGGTAGTAGCG, ${ }^{25}$ COL1A1-Forward: ACATGTTCAGCTTTGTGGACC, and COL1-A1-Reverse: AGGTTTCCACGTCTCACCAT, RUNX-2-Forward: CCGAGCTACGAAATGCCTCT, and RUNX-2-Reverse: $\quad$ GGACCGTCCACTGTCACTTT. ${ }^{18}$ Each experiment was repeated 3 times. Fold change was calculated using $\mathrm{Ct}(2-\Delta \Delta \mathrm{Ct}){ }^{26}$

\section{Western Blot Analysis}

Proteins of treated cells were extracted using RIPA buffer and quantified by a BCA kit (Promega). Same amounts of denatured proteins were separated by SDS-PAGE and transferred to PVDF membranes. Membranes were blocked with $6 \%$ non-fat milk and incubated with primary antibodies (anti-RUNX-2, anti-Col1 A1 or anti-GAPDH) for 12 hours at $4{ }^{\circ} \mathrm{C}$, followed by incubation with HRP conjugated secondary antibodies for 1 hour and visualization with an ECL kit. Image-Pro 6.0 was used for image analysis (Media Cybernetics, USA) 


\section{Cell Viability Assay Using WST-8 \\ Reduction Assay Kit}

mMSCs were cultured in a 96-well plate $\left(1.5 \times 10^{3}\right.$ cells/ well) for 24 hours. Culture medium was replaced with reagents and incubated for 2 days. WST- 8 was loaded and incubated for 2 hours before measuring optical density at $450 \mathrm{~nm}$ by a microplate reader.

\section{Construction and Identification of Rat Model of Osteoporosis}

Fifty 6-month-old female Sprague Dawley (SD) rats (body weight: $300-350$ grams) were used for this study. All rats were randomly divided into five groups $(\mathrm{n}=10)$ : PBS, Ale, EVs, Ale-EVs and Control groups. Before surgery, all rats were fasted for 8 hours and anesthetized by intraperitoneal (IP) injection of sodium pentobarbital $(50 \mathrm{mg} / \mathrm{kg})$. The skin around the midline of the abdomen was shaved and disinfected. Linear incisions were made on bilateral skin of lumbar vertebrae. Bluntly dissecting the muscles and the peritoneum were bluntly dissected to expose the ovary. Then, bilateral ovaries together with some fat tissue were gently removed from rats in PBS group, Ale group, EVs group and Ale-EVs groups to establish the osteoporosis model, while the rats in the Control group only removed some adipose tissue around the ovary as controls. Finally, the tissue was repositioned, the abdominal muscle tissue and skin were sutured carefully, and penicillin was injected $(40,000 \mathrm{IU} / \mathrm{mL}, 1 \mathrm{~mL} / \mathrm{kg})$ for 3 consecutive days. Twelve weeks after surgery, 3 rats were selected from each group to evaluated the establishment of osteoporosis model by micro-CT analysis of distal femur and detection of serum estrogen concentration.

\section{Micro-CT Analysis}

A micro-CT ( $\mu$ CT50; Scanco Medical, Brüttisellen, Switzerland) was used for this study. Briefly, 100 slices with a voxel size of $10 \mu \mathrm{m}$ were scanned in the region of the distal femur, beginning at the growth plate and extending proximally along the femur diaphysis. Eighty continuous slices beginning at $0.1 \mathrm{~mm}$ from the most proximal aspect of the growth plate in which both condyles were no longer visible were selected for analysis. After 3D reconstruction, bone mineral density (BMD), bone volume fraction (BV/TV), trabecular number (Tb.N), trabecular thickness (Tb.Th) and trabecular separation (Tb.Sp) were automatically determined to confirm the osteoporotic model, while the BMD and BV/TV values in the defect regions were used to evaluate new bone formation using the auxiliary software (Scanco).

\section{Statistics}

Data are presented as Mean $\pm \mathrm{SD}$. All statistical analyses were performed using GraphPad Prism 8.0.2 (GraphPad Software, San Diego, CA, USA). Statistical differences between two groups were analyzed using Student's $t$-test. Differences among multiple groups were analyzed using ANOVA. A P value less than 0.05 was defined as statistically significance.

\section{Results}

\section{Synthesis and Characterization of ALE-EVs}

EVs were isolated from culture supernatants of BMMSCs. N3-Ale and EVs were coupled using copperfree "click chemistry" approach (Figure 1A). First, in order to let EVs have a DBCO functional group, the compound DBCO-PEG-NHS was decorated on the surface of EVs through reaction of NHS ester functional group with amine group (NH2) of EVs membrane protein. Then, N3-Ale (Supplementary Figures S1 and

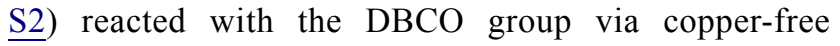
"click chemistry". To confirm the successful conjugation of the N3 with the DBCO, Cy5.5-N3 was used. Next, we captured the Cy5.5-conjugated EVs by Dynabeads ${ }^{\circledR}$ with CD63 antibody and measured fluorescence signal of the beads by confocal fluorescence microscopy. Results showed that the beads with Cy5.5EVs had strong red fluorescence signal, but the beads with DBCO-EVs has no fluorescence signal (Figure 1B). This indicated that Cy5.5-N3 successfully bound to EVs membrane. N3-Ale was bound to EVs membrane using the same method. Ale-EVs were spherical and intact under TEM (Figure 1C). Dynamic light scattering (DLS) showed that the average diameter of Ale-EVs was $53.7 \mathrm{~nm}$ (Figure 1D).

\section{Bone Targeting of Ale-EVs}

Ale has a high affinity with hydroxyapatite (HA), which is the main component of bone matrix. To determine whether AleEVs binds HA beads, Ale-EVs stained with DiD (red fluorescence) were incubated with the hydroxyapatite beads. EVs stained with DiD was used as a control. As expected, flow cytometry results showed that the HA beads with Ale-EVs 
A

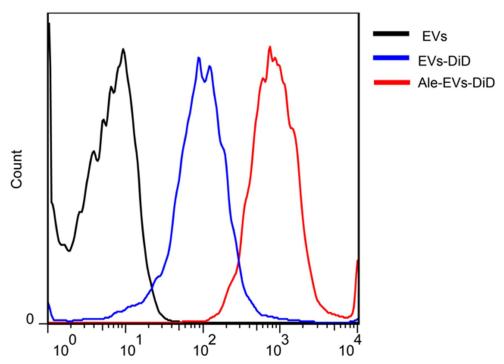

B

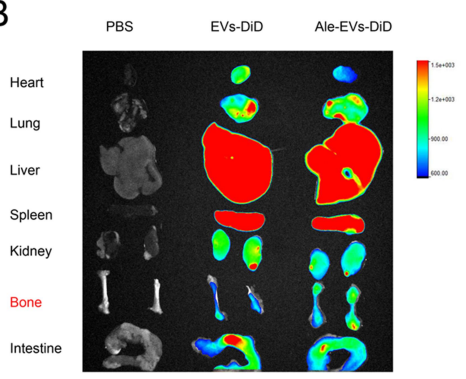

C

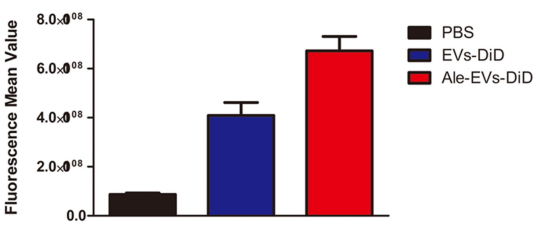

Figure 2 Bone targeting of Ale-EVs. (A) Binding of Ale-EVs-DiD with HA beads detected by flow cytometry. Ale-EVs or EVs were loaded with DiD and then incubated with the HA beads at RT for 30 minutes. The result showed that the fluorescent signal was relatively stronger in HA beads incubated with Ale-EVs-DiD. (B) Ex vivo fluorescent images of major mouse organs at 6 hour after injection with $150 \mu g$ of Ale-EVs-DiD, EVs-DiD or PBS. In Ale-EVs-DiD group, bone tissues had stronger fluorescence signals. In EVs-DiD group, bone tissues had relatively weaker fluorescence signal. (C) Fluorescence quantification. All data presented as means \pm SE, $n=3$ per group.

Abbreviations: Ale, alendronate; EVs, extracellular vesicles; HA, hydroxyapatite; DiD, I,I'-dioctadecyl-3,3,3',3'-tetramethylindodicarbocyanine perchlorate; RT, room temperature.

-DiD had a much stronger fluorescent signal than the HA beads with EVs-DiD (Figure 2A). To measure bonetargeting ability of Ale-EVs, the Ale-EVs and EVs stained by DiD were injected (iv) into nude mice (2 months old). PBS was used as control. Ale-EVs-DiD and EVs-DiD were tracked by in vivo imaging at 6 hours after injection. Ex vivo fluorescent imaging results showed that bones from Ale-EVs-DiD group had stronger fluorescent signals than that of EVs-DiD group (Figure 2B and C).

A

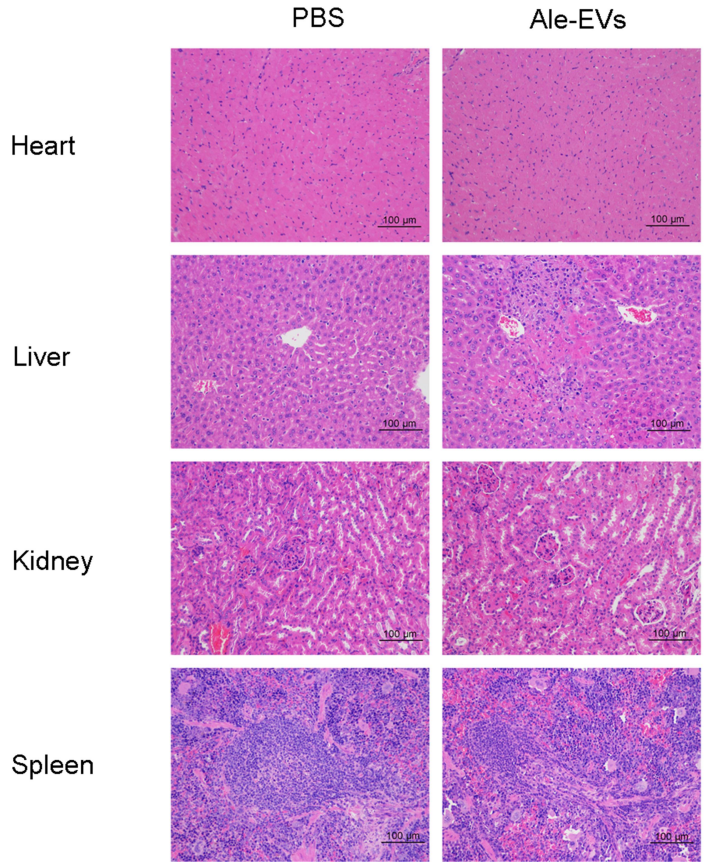

\section{Safety of Ale-EVs}

Possible heart, liver and kidney damages by Ale-EVs were monitored. BALB/c mice received PBS or Ale-EVs (150$\mu \mathrm{g}$ EVs per dose, iv) every other day for 2 weeks. Hematoxylin and eosin staining were used to monitor tissue damage. No obvious tissue damage was found (Figure 3A).

Serum levels of creatine kinase MB isoenzyme (CK$\mathrm{MB})$ and blood urea nitrogen (BUN) were also tested for heart toxicity and kidney toxicity. No significant

B

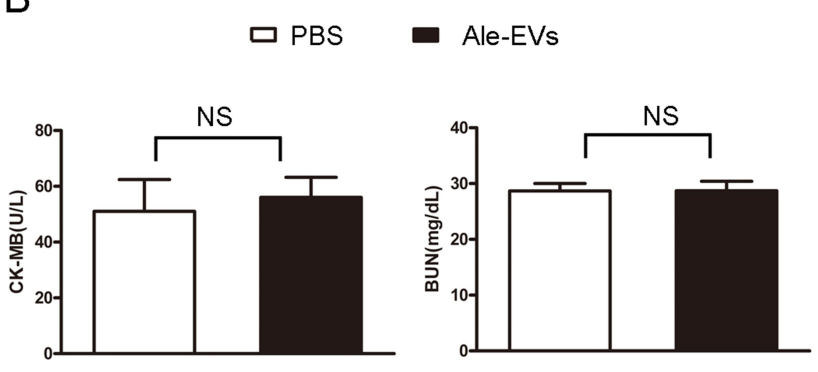

C
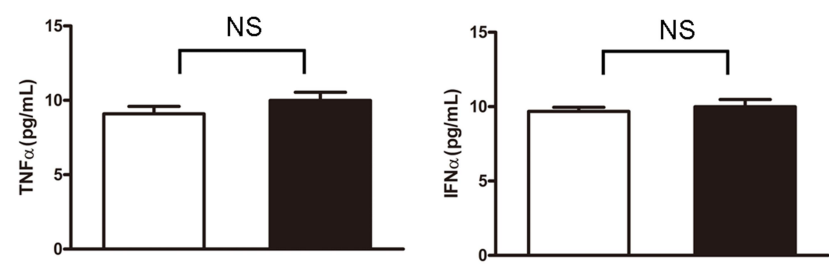

Figure 3 Safety test of Ale-EVs. (A) H\&E staining (Scale bar $=100 \mu \mathrm{m})$. (B) Levels of CK-MB and BUN. (C) Levels of TNF- $\alpha$ and INF- $\alpha$. All data presented as means \pm SE, $\mathrm{n}=6$ per group.

Abbreviations: Ale, alendronate; EVs, extracellular vesicles; CK-MB, creatine kinase-MB isoenzyme; BUN, blood urea nitrogen; NS, no significant. Difference noted between the 2 treatment groups. 
difference was found between Ale-EVs group and PBS group (Figure 3B).

To assess possible immuno-responses caused by AleEVs, levels of INF- $\alpha$ and TNF- $\alpha$ were measured. Ale-EVs did not affect INF- $\alpha$ levels (Figure 3C), indicating that Ale-EVs are not toxic for in vivo therapy.

\section{Biological Function of Ale-EVs in vitro}

After observed with no toxicity of mice treated with Ale-EVs, we assessed effects of Ale-EVs on cell growth and differentiation. Ale-EVs, EVs, Ale and PBS were incubated with mMSCs for $48 \mathrm{~h}$ followed by measurement of cell growth. Ale-EVs and EVs promoted cell growth compared with PBS (Figure 4A). Ale treatment did not affect viability.

Next, we incubated Ale-EVs, EVs, Ale and PBS with mMSCs for 7 days and 14 days, respectively. And then the early marker of osteogenic differentiation was measured by ALP activity assessment. The results showed that the OD values of Ale-EVs, EVs and Ale group were higher than the control group (Figure 4B). We also assessed the levels of bone-related mRNA and protein levels and proved that levels of COL1 and RUNX-2 treated with AleEVs and EVs were remarkably increased compared with Ale and PBS groups (Figure 4C and D).

\section{Antiosteoporotic Effects of Ale-EVs in vivo}

To investigate the potential application of Ale-Evs in OP therapy, an ovariectomy (OVX)-induced osteoporosis rat model was established. Ale-Evs were administered to OVX rats by tail vein injection. The rats were randomly separated to 4 groups $(n=4)$ to receive PBS, Ale, EVs, or Ale-EVs by tail vein injection. Rats were treated (EVs per dose, $750 \mu \mathrm{g}$, iv) twice a week for a total of 16 injections. Micro-CT results showed that bone microarchitecture and bone mass was much better in Ale-EVs group (Figure 5A and B). BV/TV values were markedly higher in Ale-EVs group (Figure 5C). Besides, trabecular thickness and trabecular number in distal femora were increased and trabecular spacing were decreased in Ale-EVs group (Figure 5D). These results suggested AleEVs promote bone regeneration in osteoporotic conditions.

\section{Discussion}

Alendronate is a bisphosphonate that binds with hydroxyapatite crystals in bone to reduce osteoclast-mediated bone resorption and reduce bone matrix destruction. ${ }^{12}$ It has some side-effects such as osteonecrosis of the jaw, ${ }^{27}$ oral mucosa ulcerations, ${ }^{28}$ synovitis, ${ }^{29}$ arthritis ${ }^{30}$ and GI symptoms. ${ }^{12}$ Using nano-carriers as drug delivery system (DDS) has been shown to prolong the drug cycle time and
A

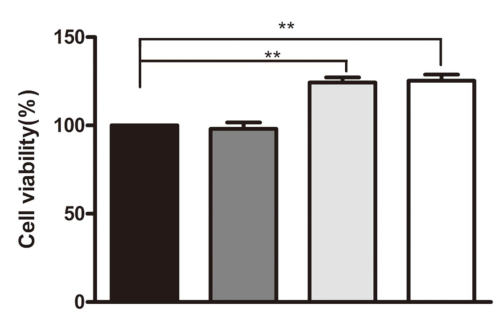

C

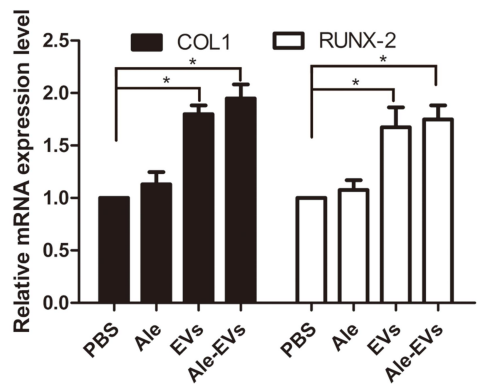

B

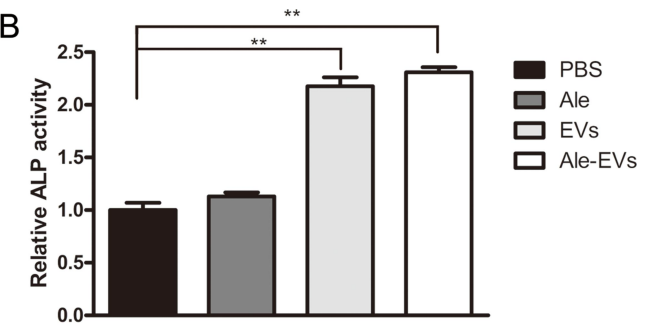

D

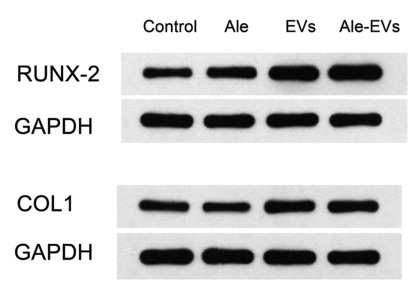

Figure 4 Antiosteoporosis efficacy of Ale-EVs in vitro. MMSCs were incubated with PBS, Ale, EVs and Ale-EVs (300 $\mu \mathrm{g} / \mathrm{mL}$, equal to free EVs). After 48 hours, Ale-EVs and EVs promoted cells growth. (A) ALP assay on Day 14 post-treatment. Ale-EVs and EVs significantly promoted cells ALP activity. (B) RUNX-2 and COLI expression at mRNA level (C) and protein level (D). Ale-EVs and EVs promoted expression of RUNX-2 and COLI remarkably. All data presented as means $\pm S E$, $n=6$ per group. *P $<$ $0.05 ; * * P<0.01$.

Abbreviations: Ale, alendronate; EVs, extracellular vesicles; ALP, alkaline phosphatase. 
A

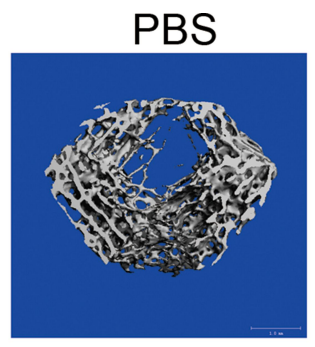

B

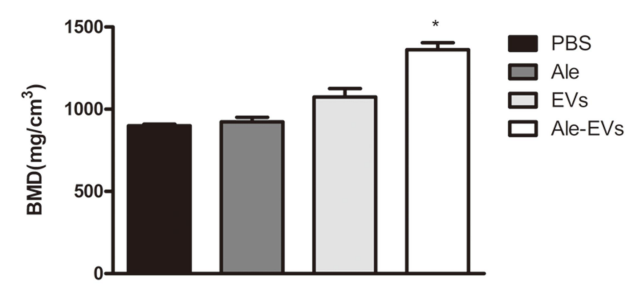

Ale

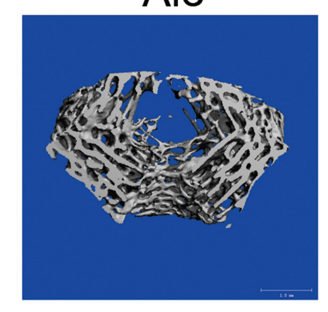

Ale-EVs

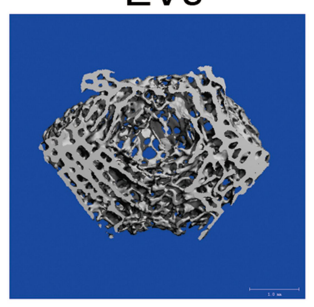

C

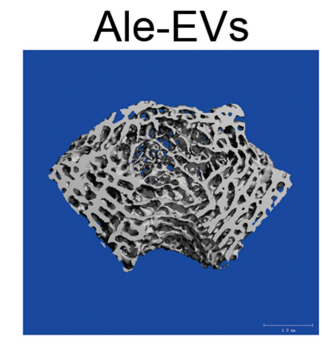

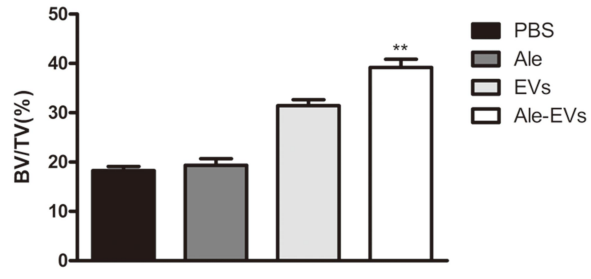

D
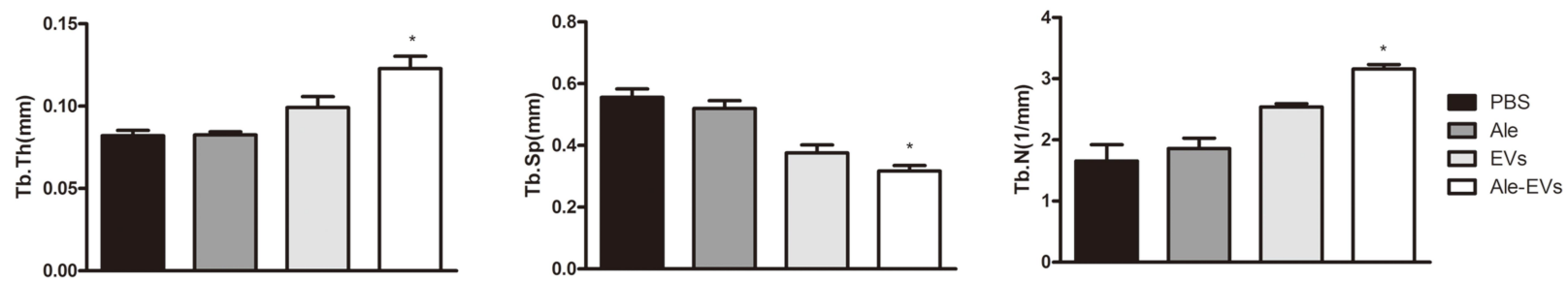

Figure 5 Antiosteoporosis efficacy of Ale-EVs in vivo. (A) 3-D trabecular architecture (bars I mm). (B) BMD in distal femora. (C) BV/TV in distal femora. (D) Tb.Th, Tb.Sp and Tb.N in distal femora. All data presented as means $\pm \mathrm{SE}, \mathrm{n}=6$ per group. $* \mathrm{P}<0.05$; $* * \mathrm{P}<0.0 \mathrm{I}$.

Abbreviations: BMD, bone-mineral density; BV, bone volume; TV, total volume; Tb.Th, trabecular thickness; Tb.Sp, Tb spacing; Tb.N, Tb number.

reduce the dosage and side effects of alendronate drugs. For instance, peptide-based nanoparticles ${ }^{31}$ and hydrogels $^{32}$ are currently being used for drug delivery due to their ease of tunability. In this study, we used a similar method to generate Ale-EVs nanoparticle systems. Ale was coupled to the surface of EVs to serve as a bone-targeting ligand. Because of the low content of Ale coupled to the surface of EVs, its side effects were greatly reduced. The anti-osteoporosis effect of free Ale was not obvious due to the low content (Figure 5). It could be possible to get a better anti-osteoporosis effect of AleEVs if the coupling amount of Ale was increased.

It has been reported that MSC-derived exosomes and microparticles could stimulate the repair and regeneration of tissues such as kidney, heart, and bone. ${ }^{33-37}$ Exosomes contain functional mRNAs, IncRNA, tRNA and miRNAs, as well as proteins. In addition, these contents play a positive role in osteogenic differentiation and proliferation. It is reported that the osteoporotic phenotype of Fasdeficient MRL/lpr mice can be rescued by MSC-derived exosomal Fas protein. ${ }^{38}$ Moreover, microRNAs in MSCderived exosomes have been shown to play an important role in MSC-mediated angiogenesis. ${ }^{39}$ Data also indicated that exosomes derived from mesenchymal stem cells can promote cell proliferation, osteogenic differentiation and bone formation both in vitro and in vivo. ${ }^{40,41}$ Our study indicated that mMSCs-derived EVs promoted cell growth and triggered osteogenic differentiation in vitro (Figure 4). It indicated that the MSCs-derived EVs had the promising applications in reducing the side effects of Ale. In our study, alendronate was conjugated to the membrane surface protein of EVs through "click chemistry" approach (Figure 1A), and results showed that EVs bone-targeting ability was improved (Figure 2). By this way, we successfully combined the advantages of MSCs-derived EVs and alendronate for osteoporosis therapy (Figure 1A). As EVs are widely used for drug delivery, ${ }^{42}$ it is important to confer EVs specific tissue targeting ability. We provided a new method to modify EVs' surface membrane for targeting ligand modification. The earliest targeted delivery strategies for EVs were mainly based on the fusion of targeted ligands with EV membrane proteins such as lamp2b. ${ }^{43-45}$ But this strategy is time-consuming and Some Lamp2b fusion targeting ligands are easy to 
degrade, thus affecting their function. ${ }^{46}$ Along with these, another non-covalent modification strategies are also being studied such as multivalent electrostatic interactions, ${ }^{47,48}$ ligand-receptor interactions, ${ }^{49}$ hydrophobic interactions, ${ }^{50}$ aptamer based surface modification ${ }^{51}$ and modification by anchoring CP05 peptide. ${ }^{52,53}$ This method of non-covalent bond modification is a challenge to the stability between ligands and EVs in a complex environment in vivo. The covalent binding method through click chemistry is effective and will not cause EVs membrane destruction or EVs aggregation due to reaction conditions. Tang team also prepared the functional EVs of RGE peptides by clicking the chemical reaction. ${ }^{54}$ In addition, this method can also be used to load small molecular drugs instead of the previous inefficient methods method such as electroporation, extrusion, sonication, incubation, freeze-thaw. ${ }^{55,56}$

In addition, Ale-EVs could be used as a bone-target delivery system. We can enrich mMSCs-derived EVs with mRNAs or proteins to treat osteoporosis by genetic engineering technology, ${ }^{57-59}$ and strengthen the antiosteoporosis effect of Ale-EVs. Besides, we also could load other drugs such as nucleic acid drugs, ${ }^{36,60}$ small molecule drugs ${ }^{61}$ into the target-delivery system to achieve the purpose of combined treatment of osteoporosis.

Although we successfully assessed the bone targeting ability of Ale-EVs, there are a few issues that need to be addressed in future studies. First, due to the complexity of EVs and the lack of detectable markers on Ale, we cannot directly prove that Ale is coupled to the surface of EVs, nor can we calculate the amount of coupling. Therefore, we used Cy5.5-N3 to couple to the surface of EVs, it is easy to be detected because of the special fluorescent signal of Cy5.5. It proved that the compounds containing N3 groups could effectively couple with EVs-DBCO (Figure 1A and B). This result indirectly proved that Ale-N3 could couple to the surface of EVs-DBCO. Extra experiments are needed to directly prove the method of surface coupling between Ale-N3 and EVs-DBCO, and to quantify Ale on the EVs surface. Second, alendronate is known to enhance bone quality by inhibiting osteoclasts. In our study, the Ale of AleEVs is used as a bone-targeting ligand to give EVs bone targeting ability. However, whether Ale-EVs has an inhibitory effect on osteoclasts needs to be further studied.

\section{Conclusion}

Taken together, Ale-N3 was used to modify mMSCsderived EVs to generate Ale-EVs. Ale-EVs showed a high affinity for bone with low toxic effect. Therefore, Ale-EVs could be used in the treatment of osteoporosis.

\section{Data Sharing Statement}

All data are included in this paper.

\section{Ethics Approval and Consent to Participate}

Approval for the animal experiments was obtained in advance from the Animal Ethics Committee of Guangzhou Curegenix Ltd on Animal Experiments (ethical permit YSDW201812052) and all experiments in this work conform to the regulatory standards of this approval.

\section{Consent for Publication}

All authors agree to be published.

\section{Author Contributions}

All authors made a significant contribution to the work reported, whether that is in the conception, study design, execution, acquisition of data, analysis and interpretation, or in all these areas; took part in drafting, revising or critically reviewing the article; gave final approval of the version to be published; have agreed on the journal to which the article has been submitted; and agree to be accountable for all aspects of the work.

\section{Funding}

This work was supported by Guangdong Natural Science Funds (2018A030313614); The Fundamental Research Funds for the Central Universities (21619407).

\section{Disclosure}

The authors declare no potential conflict of interest.

\section{References}

1. Lorentzon M. Treating osteoporosis to prevent fractures: current concepts and future developments. J Intern Med. 2019;285(4):381-394. doi:10.1111/joim. 12873

2. Tarantino U, Cerocchi I, Scialdoni A, et al. Bone healing and osteoporosis. Aging Clin Exp Res. 2011;23(2):62-64.

3. Temmerman A, Rasmusson L, Kübler A, Thor A, Merheb J, Quirynen M. A prospective, controlled, multicenter study to evaluate the clinical outcome of implant treatment in women with osteoporosis/ osteopenia: 5-year results. J Dent Res. 2019;98(1):84-90. doi:10.1177/ 0022034518798804

4. Merheb J, Temmerman A, Rasmusson L, Kübler A, Thor A, Quirynen M. Influence of skeletal and local bone density on dental implant stability in patients with osteoporosis. Clin Implant Dent Relat Res. 2016;18(2):253-260. doi:10.1111/cid.12290 
5. Wagner F, Schuder K, Hof M, Heuberer S, Seemann R, Dvorak G. Does osteoporosis influence the marginal peri-implant bone level in female patients? A cross-sectional study in a matched collective. Clin Implant Dent Relat Res. 2017;19(4):616-623. doi:10.1111/cid.12493

6. Lewis JR, Schousboe JT, Prince RL. Romosozumab versus alendronate and fracture risk in women with osteoporosis. $N$ Engl J Med. 2018;378(2):194-195.

7. Fink HA, MacDonald R, Forte ML, et al. Long-term drug therapy and drug discontinuations and holidays for osteoporosis fracture prevention: a systematic review. Ann Intern Med. 2019;171(1):37. doi:10.7326/M19-0533

8. Naot D, Musson DS, Cornish J. The activity of peptides of the calcitonin family in bone. Physiol Rev. 2018;99(1):781-805. doi:10.1152/physrev.00066.2017

9. Dempster DW, Zhou H, Ruff VA, Melby TE, Alam J, Taylor KA. Longitudinal effects of teriparatide or zoledronic acid on bone modeling-and remodeling-based formation in the SHOTZ study. $J$ Bone Miner Res. 2018;33(4):627-633. doi:10.1002/jbmr.3350

10. Khan M, Cheung AM, Khan AA. Drug-related adverse events of osteoporosis therapy. Endocrinol Metab Clin. 2017;46(1):181-192. doi:10.1016/j.ecl.2016.09.009

11. Guan M, Yao W, Liu R, et al. Directing mesenchymal stem cells to bone to augment bone formation and increase bone mass. Nat Med. 2012;18(3):456. doi:10.1038/nm.2665

12. Parker LRW, Preuss CV. Alendronate. In: StatPearls [Internet]. StatPearls Publishing; 2018.

13. Théry C, Zitvogel L, Amigorena S. Exosomes: composition, biogenesis and function. Nat Rev Immunol. 2002;2(8):569. doi:10.1038/ nri855

14. Villasante A, Marturano-Kruik A, Ambati SR, et al. Recapitulating the size and cargo of tumor exosomes in a tissue-engineered model. Theranostics. 2016;6(8):1119. doi:10.7150/thno.13944

15. Ramirez MI, Amorim MG, Gadelha C, et al. Technical challenges of working with extracellular vesicles. Nanoscale. 2018;10(3):881-906. doi:10.1039/C7NR08360B

16. Zhu L-P, Tian T, Wang J-Y, et al. Hypoxia-elicited mesenchymal stem cell-derived exosomes facilitates cardiac repair through miR-125b-mediated prevention of cell death in myocardial infarction. Theranostics. 2018;8(22):6163. doi:10.7150/thno.28021

17. $\mathrm{Hu} \mathrm{Y,} \mathrm{Xu} \mathrm{R,} \mathrm{Chen} \mathrm{C-Y,} \mathrm{et} \mathrm{al.} \mathrm{Extracellular} \mathrm{vesicles} \mathrm{from} \mathrm{human}$ umbilical cord blood ameliorate bone loss in senile osteoporotic mice. Metabolism. 2019;95:93-101. doi:10.1016/j.metabol.2019.01.009

18. Qi X, Zhang J, Yuan H, et al. Exosomes secreted by human-induced pluripotent stem cell-derived mesenchymal stem cells repair critical-sized bone defects through enhanced angiogenesis and osteogenesis in osteoporotic rats. Int J Biol Sci. 2016;12(7):836.

19. Kacprzak K, Skiera I, Piasecka M, Paryzek Z. Alkaloids and isoprenoids modification by copper (I)-catalyzed Huisgen 1, 3-dipolar cycloaddition (click chemistry): toward new functions and molecular architectures. Chem Rev. 2016;116(10):5689-5743. doi:10.1021/acs. chemrev. 5 b00302

20. Thirumurugan P, Matosiuk D, Jozwiak K. Click chemistry for drug development and diverse chemical-biology applications. Chem Rev. 2013;113(7):4905-4979. doi:10.1021/cr200409f

21. Park JY, Song MG, Kim WH, et al. Versatile and finely tuned albumin nanoplatform based on click chemistry. Theranostics. 2019;9(12):3398. doi:10.7150/thno.33143

22. Stefanucci A, Lei W, Pieretti S, et al. On resin click-chemistrymediated synthesis of novel enkephalin analogues with potent anti-nociceptive activity. Sci Rep. 2019;9(1):5771. doi:10.1038/ s41598-019-42289-5

23. Lee HJ, Fernandes-Cunha GM, Putra I, Koh W-G, Myung D. Tethering growth factors to collagen surfaces using copper-free click chemistry: surface characterization and in vitro biological response. ACS Appl Mater Interfaces. 2017;9(28):23389-23399. doi:10.1021/acsami.7b05262
24. Schwarzenböck C, Nelson PJ, Huss R, Rieger B. Synthesis of next generation dual-responsive cross-linked nanoparticles and their application to anti-cancer drug delivery. Nanoscale. 2018;10 (34):16062-16068. doi:10.1039/C8NR04760J

25. Zhang H, Sun Z, Wei W, et al. Identification of serum microRNA biomarkers for tuberculosis using RNA-seq. PLoS One. 2014;9(2): e88909.

26. Yuan JS, Reed A, Chen F, Stewart CN. Statistical analysis of real-time PCR data. BMC Bioinform. 2006;7(1):85. doi:10.1186/ 1471-2105-7-85

27. Reid IR, Bolland MJ, Grey AB Is bisphosphonate-associated osteonecrosis of the jaw caused by soft tissue toxicity? Elsevier; 2007:318-320.

28. Gonzalez-Moles MA, Bagan-Sebastian JV. Alendronate-related oral mucosa ulcerations. J Oral Pathol Med. 2000;29(10):514-518. doi:10.1034/j.1600-0714.2000.291006.x

29. Jones DPG, Savage RL, Highton J. Alendronate-induced synovitis. J Rheumatol. 2008;35(3):537-538.

30. Diaz-Borjon A, Seyler TM, Chen NL, Lim SS. Bisphosphonateassociated arthritis. J Clin Rheumatol. 2006;12(3):131-133.

31. Chan T. Advancement of peptide nanobiotechnology via emerging microfluidic technology. Micromachines. 2019;10(10):627. doi:10.3390/mi10100627

32. Chan KH, Lee WH, Ni M, et al. C-terminal residue of ultrashort peptides impacts on molecular self-assembly, hydrogelation, and interaction with small-molecule drugs. Sci Rep. 2018;8:1-14. doi:10.1038/s41598-018-35431-2

33. Wang $\mathrm{R}$, Lin $\mathrm{M}$, Li L, et al. Bone marrow mesenchymal stem cell-derived exosome protects kidney against ischemia reperfusion injury in rats. Zhonghua Yi Xue Za Zhi. 2014;94 (42):3298-3303.

34. Zhang J, Guan J, Niu X, et al. Exosomes released from human induced pluripotent stem cells-derived MSCs facilitate cutaneous wound healing by promoting collagen synthesis and angiogenesis. J Transl Med. 2015;13(1):49. doi:10.1186/s12967015-0417-0

35. Narayanan R, Huang C-C, Ravindran S. Hijacking the cellular mail: exosome mediated differentiation of mesenchymal stem cells. Stem Cells Int. 2016;2016:1-11. doi:10.1155/2016/3808674

36. Nie H, Xie X, Zhang D, et al. Use of lung-specific exosomes for miRNA-126 delivery in non-small cell lung cancer. Nanoscale. 2020;12(2):877-887. doi:10.1039/C9NR09011H

37. Tao S-C, Yuan T, Zhang Y-L, Yin W-J, Guo S-C, Zhang C-Q. Exosomes derived from miR-140-5p-overexpressing human synovial mesenchymal stem cells enhance cartilage tissue regeneration and prevent osteoarthritis of the knee in a rat model. Theranostics. 2017;7 (1): 180. doi:10.7150/thno. 17133

38. Liu S, Liu D, Chen C, et al. MSC transplantation improves osteopenia via epigenetic regulation of notch signaling in lupus. Cell Metab. 2015;22(4):606-618. doi:10.1016/j.cmet.2015.08.018

39. Gong M, Yu B, Wang J, et al. Mesenchymal stem cells release exosomes that transfer miRNAs to endothelial cells and promote angiogenesis. Oncotarget. 2017;8(28):45200-45212. doi:10.18632/ oncotarget. 16778

40. Jia Y, Qiu S, Xu J, Kang Q, Chai Y. Exosomes secreted by young mesenchymal stem cells promote new bone formation during distraction osteogenesis in older rats. Calcif Tissue Int. 2020;106 (5):509-517. doi:10.1007/s00223-019-00656-4

41. Qi X, Zhang J, Yuan H, et al. Exosomes secreted by human-induced pluripotent stem cell-derived mesenchymal stem cells repair critical-sized bone defects through enhanced angiogenesis and osteogenesis in osteoporotic rats. Int J Biol Sci. 2016;12(7):836-849. doi:10.7150/ijbs. 14809

42. Vader P, Mol EA, Pasterkamp G, Schiffelers RM. Extracellular vesicles for drug delivery. Adv Drug Deliver Rev. 2016;106:148-156. doi:10.1016/j.addr.2016.02.006 
43. Alvarez-Erviti L, Seow Y, Yin H, Betts C, Lakhal S, Wood M. Delivery of siRNA to the mouse brain by systemic injection of targeted exosomes. Nat Biotechnol. 2011;29(4):341-345. doi: $10.1038 /$ nbt. 1807

44. Cooper J, Wiklander P, Nordin J, et al. Systemic exosomal siRNA delivery reduced alpha-synuclein aggregates in brains of transgenic mice. Mov Disord. 2014;29(12):1476-1485. doi:10.1002/mds.25978

45. Tian Y, Li S, Song J, et al. A doxorubicin delivery platform using engineered natural membrane vesicle exosomes for targeted tumor therapy. Biomaterials. 2014;35(7):2383-2390. doi:10.1016/j. biomaterials.2013.11.083

46. Hung M, Leonard J. Stabilization of exosome-targeting peptides via engineered glycosylation. J Biol Chem. 2015;290(13):8166-8172. doi:10.1074/jbc.M114.621383

47. Tamura R, Uemoto S, Tabata Y. Augmented liver targeting of exosomes by surface modification with cationized pullulan. Acta Biomater. 2017;57:274-284. doi:10.1016/j.actbio.2017.05.013

48. Armstrong J, Holme M, Stevens M. Re-engineering extracellular vesicles as smart nanoscale therapeutics. ACS Nano. 2017;11 (1):69-83. doi:10.1021/acsnano.6b07607

49. Wang J, Li W, Zhang L, et al. Chemically edited exosomes with dual ligand purified by microfluidic device for active targeted drug delivery to tumor cells. ACS Appl Mater Interfaces. 2017;9 (33):27441-27452. doi:10.1021/acsami.7b06464

50. Sato Y, Umezaki K, Sawada S, et al. Engineering hybrid exosomes by membrane fusion with liposomes. Sci Rep. 2016;6:21933. doi: $10.1038 /$ srep21933

51. Wan S, Zhang L, Wang S, et al. Molecular recognition-based DNA nanoassemblies on the surfaces of nanosized exosomes. J Am Chem Soc. 2017;139(15):5289-5292. doi:10.1021/jacs.7b00319

52. Gao X, Zhao J, Han G, et al. Effective dystrophin restoration by a novel muscle-homing peptide-morpholino conjugate in dystrophin-deficient MDX mice. Mol Ther. 2014;22(7):1333-1341. doi: $10.1038 / \mathrm{mt} .2014 .63$
53. Kooijmans S, Aleza C, Roffler S, van Solinge W, Vader P, Schiffelers R. Display of GPI-anchored anti-EGFR nanobodies on extracellular vesicles promotes tumour cell targeting. J Extracell Vesicles. 2016;5:31053. doi:10.3402/jev.v5.31053

54. Jia G, Han Y, An Y, et al. NRP-1 targeted and cargo-loaded exosomes facilitate simultaneous imaging and therapy of glioma in vitro and in vivo. Biomaterials. 2018;178:302-316. doi:10.1016/j. biomaterials.2018.06.029

55. Haney M, Klyachko N, Zhao Y, et al. Exosomes as drug delivery vehicles for Parkinson's disease therapy. $J$ Control Release. 2015;207:18-30. doi:10.1016/j.jconrel.2015.03.033

56. Kooijmans S, Stremersch S, Braeckmans K, et al. Electroporationinduced siRNA precipitation obscures the efficiency of siRNA loading into extracellular vesicles. $J$ Control Release. 2013;172 (1):229-238. doi:10.1016/j.jconrel.2013.08.014

57. Zhuang M, Chen X, Du D, et al. SPION decorated exosome delivery of TNF- $\alpha$ to cancer cell membranes through magnetism. Nanoscale. 2020;12(1):173-188. doi:10.1039/C9NR05865F

58. Yang Z, Shi J, Xie J, et al. Large-scale generation of functional mRNA-encapsulating exosomes via cellular nanoporation. Nat Biomed Eng. 2020;4(1):69-83. doi:10.1038/s41551-019-0485-1

59. Hall J, Prabhakar S, Balaj L, Lai CP, Cerione RA, Breakefield XO. Delivery of therapeutic proteins via extracellular vesicles: review and potential treatments for Parkinson's disease, glioma, and schwannoma. Cell Mol Neurobiol. 2016;36(3):417-427. doi:10.1007/ s10571-015-0309-0

60. Zhang G, Guo B, Wu H, et al. A delivery system targeting bone formation surfaces to facilitate RNAi-based anabolic therapy. Nat Med. 2012;18(2):307. doi:10.1038/nm.2617

61. Higdon K, Scott A, Tucci M, et al. The use of estrogen, DHEA, and diosgenin in a sustained delivery setting as a novel treatment approach for osteoporosis in the ovariectomized adult rat model. Biomed Sci Instrum. 2001;37:281-286.
International Journal of Nanomedicine

\section{Publish your work in this journal}

The International Journal of Nanomedicine is an international, peerreviewed journal focusing on the application of nanotechnology in diagnostics, therapeutics, and drug delivery systems throughout the biomedical field. This journal is indexed on PubMed Central, MedLine, CAS, SciSearch ${ }^{\circledR}$, Current Contents ${ }^{\circledR} /$ Clinical Medicine,
Journal Citation Reports/Science Edition, EMBase, Scopus and the Elsevier Bibliographic databases. The manuscript management system is completely online and includes a very quick and fair peer-review system, which is all easy to use. Visit http://www.dovepress.com/ testimonials.php to read real quotes from published authors. 